\title{
Mapping the perception of "complex systems" across educational levels through cognitive network science
}

\author{
Massimo Stella \\ Complex Science Consulting, Italy \\ Correspondence: massimo.stella@inbox.com
}

\begin{abstract}
Despite recent efforts promoting complexity science across different educational contexts, there is little literature about how school students perceive complex systems. This research report aims to quantify the current perception of "complex systems" among 159 Italian high school students, providing a data-informed map of the general attitude and knowledge structure towards complexity through tools from cognitive network science. Adopting the framework of forma mentis networks, i.e. conceptual networks where words are related by memory recall patterns and labelled according to their positive/negative/neutral sentiment, the students' mindset or forma mentis towards "complex systems" was reconstructed and compared to the mindset of 59 international postgraduate researchers working on complexity topics. Despite studying multiple scientific disciplines at the same time, students perceived complexity as an abstract and negative entity, strongly associated to "complicated" and "difficult" whereas researchers identified complexity as a positive concept, with a stronger STEM-oriented, multidisciplinary connotation towards mathematics, physics, biology and other scientific disciplines. This comparison was discussed in light of relevant literature about silo mentality in education. Mindset reconstruction through forma mentis networks opens novel ways for quantifying current perceptions of "complexity science" in mainstream educational curricula, suggesting key challenges for developing complexity education through the mindsets of complexity researchers.
\end{abstract}

Keywords: Complex networks, Education, Complex Systems, Cognitive Network Science.

\section{INTRODUCTION}

Complexity science is an umbrella encompassing multidisciplinary and transdisciplinary approaches to problems that could not be solved by using reductionism (Morin, 2007). The epistemologist Edgar Morin described a system as "complex" when the whole is more than the sum of its parts: in order to describe the behavior of a complex system, microscopic knowledge about how its components work individually is not 
enough. Additional knowledge about connections and interactions between such components should be obtained as well. The last few decades have seen a drastic increase in the number of publications adopting complexity science approaches, with important applications across a wide variety of qualitative and quantitative disciplines (cf. Downey, 2018).

This spurt of complex systems approaches has recently motivated the promotion of complexity science tools and methodologies beyond academia, mainly through outreach and educational initiatives teaching complexity science already at the school level (Van der Cingel, 2018). Some notable examples are the Santa Fe Institute's Complexity Explorer platform, that is open to students of different levels and backgrounds (Lizier et al., 2018), the Network Science Education initiative (Cramer et al., 2018), promoting the culture of complex networks already in high schools, the Lecturers Without Borders project (https://scied.network/, last accessed: 17/12/2019), promoting complexity-focused lectures and content internationally, and other literacy projects like Complexity Explained (De Domenico et al., 2019), a booklet written by complexity researchers and introducing basic concepts of complexity science.

Despite the increasing interest in promoting the culture of complex systems, approaches identifying the current perception of non-college students towards complexity science represent an important gap (Lizier et al., 2018). Addressing this gap would provide relevant data about critically negative or positive attitudes towards complexity, thus offering grounding for designing appropriate and effective outreach strategies. This research report aims to address this issue by adopting cognitive network science (Siew et al., 2019), a branch of network science that understands the cognitive structure of mindsets in terms of conceptual associations and their impact on perception and cognition. Over the last decade, cognitive network science approaches have been successful in understanding and predicting a wide variety of cognitive processes such as modelling early word learning, predicting creativity levels or identifying patterns of knowledge structuring and search (cf. Siew et al., 2019). The silver lining of such approaches is that knowledge as represented in the human mind is highly structured and this organisation influences the way words are recalled, pronounced, confused, identified or, more in general, processed through language (Aitchison, 2012; Siew, 2019). Identifying patterns of language associations can therefore be highly informative to mindset structure and knowledge processing in case the right cognitive methods and tools are adopted.

The recent framework of forma mentis networks used in this study represents an approach to mindset reconstruction by combining methods from network science and psycholinguistics (Stella et al., 2019). Forma mentis networks (FMN) are complex networks where nodes represent words and are linked by patterns of memory recall in a behavioral task known as free association game (De Deyne et al., 2013), e.g. reading "complex" makes 
an individual think of "system", motivating a link "complex - system". In addition to this semantic memory structure, forma mentis networks capture also affect (Posner et al., 2005), by having nodes/words labelled according to the main positive/negative/neutral sentiment they evoke in individuals when read. Once built, these networks can be analyzed through network science metrics in order to understand how the associative and emotional organisation of concepts reflects a mindset (forma mentis in Latin), i.e. the ways that several specific conceptual stances are perceived and interrelated. In comparison to questionnaires, the psycholinguistic nature of conceptual associations provides forma mentis networks with a higher flexibility, where stances/mindsets can emerge directly from the mental associations provided by participants and do not have to be hard-coded already within the initial set of cues. In this way, unexpected negative/positive stances around widely different topics (e.g. "maths", "nursing", "art", "fashion") can be observed together, interconnected appropriately and even tested for potential changes over time (see also Stella \& Zaytseva, 2020).

This work uses forma mentis networks as a mapping tool, accessing the microscopic details of how high school students and international researchers in complexity science perceive and associate the concepts of "complex" and "system". The cognitive data and methodology behind such cognitive mapping are explained in the Methods section. The Results section outlines discordant perceptions between students and researchers, that are related to the relevant literature in education research and cognitive science in the Discussion section.

\section{METHOD}

\section{Cognitive data}

The work reported here uses cognitive data gathered, assembled and processed in a previous investigation (cf. Stella et al., 2019). The authors reconstructed the mindset towards STEM subjects in two groups: one including 159 Italian high school students selected from 8 different and unrelated classes in three different schools and 59 international postgraduate researchers at the $\mathrm{PhD}$ and postdoc levels. As an exploratory study, Stella et al., (2019) used homogenous sampling fixing the following sociodemographic elements common to all the 159 students participating in the investigation: (i) all students had an age between 18 and 19; (ii) all students were Italians living in Salento, Italy; (iii) all students were preparing for their final exams at the end of their high-school education; (iv) all students expressed the intention to pursue more advanced studies at university level; (v) all students attended a liceo scientifico a type of 
secondary school in Italy providing an advanced educational curriculum focusing on science, technology and engineering (STEM) disciplines. The classes in which the interviewed students resided had different teachers. International researchers had in common: (i) a strong interest in complexity science; (ii) language proficiency in English comparable to level $\mathrm{C}_{2}$ (the highest) in the ESOL European framework. Researchers differed according to nationality and STEM background. For more details please refer to Stella et al., (2019).

The data was gathered through a continuous free association game (De Deyne et al., 2013), where each participant had to read a cue word and recall up to three associates as quickly as possible. Data collection was conducted in a controlled lab setting and included a total of 50 cue words per task. The free association game lasted for 10 minutes. Students performed the task in Italian whereas researchers performed the task in English. Cues were STEM-related concepts. Participants also had to attribute a score to each cue and target associates, on a Likert scale, depending on their perceived sentiment. The Likert scale, ranging from 1 (very negative) and 5 (very positive), was the same for all concepts. Concept scoring lasted for 10 minutes. Scores were processed through a statistical equivalence test aimed at classifying positive, negative and neutral concepts as perceived by each population, e.g. did all the scores attributed to "math" have a lower median than the scores attributed to all other concepts? (Stella et al., 2019). The processed FMN included both strong conceptual links, i.e. provided by at least two participants, and idiosyncratic associations, provided by single participants (for more details on this classification, commonly used in psycholinguistics, the interested reader is referred to Aitchison, 2012). Both types of connection, when aggregated at the population level, were informative of the average knowledge structure of groups and provided more nuanced, richer information (Stella et al., 2019). The data is available for research purposes: https://osf.io/xyfwg/, Last Accessed: 17/12/2019. Mathematica 11.3 was used for all the results reported here.

\section{Emotional auras as local network measures}

This study focused on the words "complex" and "system" as represented in the global forma mentis networks of students and researchers, respectively. Given the interplay between free associations and sentiment, Stella and colleagues (2019) suggest also measuring how many positive/negative/neutral concepts were associated with a word of a given sentiment as an estimate of the emotional aura of that word. Emotional auras represent concentrations of memory recall patterns that might elicit specific sentiment and, therefore, specific emotions (Posner et al., 2005). Stella et al. (2019) show how negative 
words surrounded by a negative emotional aura corresponded to concepts eliciting anxiety, i.e. a negative state of increased alertness (Posner et al., 2005).

This manuscript also performed a clique analysis. In a given network, a clique is a subgraph whose nodes are all connected with each other (Newman, 2018). The analysis here focused on the cliques including "complex", "system", and its first neighbors in either the researchers' or the students' FMNs. Randomized neighborhoods were built with words preserving their empirical degrees (i.e. the number of associations they were involved in) but being connected to each other at random. These random null models are called also configuration models (Newman, 2018). Randomising free associations disrupts their meaning and offers a reference model for estimating the probability of observing any empirical clique by chance. To this aim, 5000 configuration models of the neighborhoods of "complex" and "system" were computed in the students' and the researchers' FMNs and the probability of observing an empirical clique was estimated over this random sample.

\section{Cross-linguistic translation and global network comparison}

The students' FMN was built in Italian. Italian words were translated in English by using a consensus rule of 3 online translation services: Google Translate, Windows Bing Translator and DeepL. The most frequent translation of each Italian word across the above services was selected for the visualisation. Notice that this change did not affect network topology, which was retained as in the original Italian dataset. The translation quality was considered appropriate in $98 \%$ of the cases by the author, a native Italian speaker fluent in English. Words for which an adequate translation could not be obtained were kept in Italian.

Whereas previous approaches focused only on local network comparison (cf. Stella et al., 2019), here translations enabled a direct comparison between students' and researchers' mindsets at a global level. To this end, concepts were ranked according to their closeness centrality (Newman, 2018), capturing their relevance in the associative structure of a FMN. Closeness $c_{i}$ for node $i$ indicates the average inverse network distance $d$ between itself and its $N$ connected neighbours, in formulas:

$$
c_{i}=\frac{N}{\sum_{j} d_{i j}} .
$$

In semantic networks, the geodesic distance $d$ captures semantic relatedness (Siew et al., 2019), hence concepts with a higher closeness are on average more semantically related to their neighbors and, in this sense, more relevant for navigating the associative structure of conceptual associations. Ranking nodes through closeness and comparing these ranks directly is not appropriate in networks differing dramatically in size and connectivity, like 
the researchers' ( 1616 nodes and 3185 links) and students' ( 4483 nodes and 11728 links) FMNs. These size differences were due to the different number of participants interviewed for each network.

However, both FMNs were obtained from the same set of cue words, so that they should contain a common network component. Through the translation procedure, 928 English concepts were found in both FMNs. Considering a subgraph induced by these words on the students' and researchers' FMNs led to disconnected graphs with different link densities. Differences in connectivity were resolved by considering a minimum spanning tree (Newman, 2018) in both the largest connected subgraphs induced by the 928 common words over the students' and researchers' FMNs, an approach previously used in similitude analysis for social networks (cf. Flament, 2017). Spanning trees are subgraphs connecting all nodes in a network without ever including cycles. A minimum spanning tree employs the minimum number of links possible for a given network configuration. Notice that in this case the distance assumed for the construction of the minimum spanning tree is semantic distance between concepts, which is captured by free associations (cf. Siew et al., 2019). Mathematica 11.3 was used for computing a minimum spanning tree for the largest connected subgraphs induced by the 928 common concepts in the researchers' ( 882 nodes, 881 links) and students' ( 855 nodes, 854 links) FMNs. Having an analogous size in terms of nodes and links, these two minimum spanning trees were used for the ranking comparison, which identified closeness centrality of different concepts, including also "complex" and "system", across the mindsets of high-schoolers and researchers.

\section{RESULTS}

This section reports the local and global network results identifying how highschoolers and researchers reconstructed their perceptions of "complex" and "system".

\section{A local network comparison reflects different attitudes towards complex systems}

Figure 1 reports the forma mentis networks around "complex" and "system" as reconstructed from the full mindsets of students and researchers (Fig. 1 refers to full networks and not to spanning trees). Note that negative concepts are highlighted in red and positive ones in cyan. For students, both "complex" and "system" were perceived negatively, thus eliciting an average negative sentiment. "Complex" and "system" were also surrounded by a mixed neutral-negative emotional aura, i.e. they were associated mostly with black (neutral) or red (negative) concepts. This mixed neutral-negative connotation 
of "complex" and "system" was completely absent for researchers, who perceived and associated "complex" and "system" more positively.

Despite "complex" being presented as a cue to students with the same frequency as in researchers, the high-schoolers' responses were mostly blank in the original experiment, leading to considerably fewer associates in comparison to what researchers did. Notice that the number of free associates corresponds to network degree in here (cf. Siew et al., 2019). In cognitive science the number of free associates of a word is also a proxy for estimating semantic richness and it correlates positively with the number of contexts where a word can appear (Aitchison, 2012). Words with fewer associates tend to be related to fewer contexts and a reduced semantic richness. The larger number of associates provided by researchers indicates their enhanced awareness about the different contexts and meanings of complexity in comparison to high school students. A narrower vision of "complex" in students, in terms of lower degree and lower number of different contexts, was also confirmed by investigating specific associations, mostly relating "complex" to: (i) "difficult" and other synonyms, (ii) "building" and other architecture-related concepts, (iii) "number" and other mathematical concepts. Although lacking the architectural context, researchers associated "complex" with a wider number of contexts or clusters of words, relative to complexity theory, simulations, biology and nature, networks, dynamics, ecosystems and life, equations and mathematical tools. The edge-bundling plots (Newman, 2018) reported here clustered together words connected with each other, giving a visual impression of semantic associations and contexts.

In the students' FMN there was a strong conceptual link between "complex" and "difficulty", in addition to associations like "complex - complicated" and "complex difficult". These negative associations indicate students perceived "complex" as greatly overlapping in meaning with "difficult" and "complicated". Although synonymous to "complexity" in common language, in complex systems, complicatedness identifies a system whose behaviour requires computational effort but where no unexpected or emergent phenomena should be observed (Morin, 2007; Downey, 2018).

Complexity does not only arise from a large number of system constituents, like complicatedness does, but rather from the non-linear nature of its dynamics. A good example is the one of gas models in statistical physics (Papageorgiou et al., 2010), where the average dynamics of billions of particles can be described in detail as long as particle interactions are negligible. This leads to a system that is complicated in size but not complex. Students strongly associating "complex" with concepts related to difficulty indicates that they were not aware of the distinction between complexity and complicatedness. 


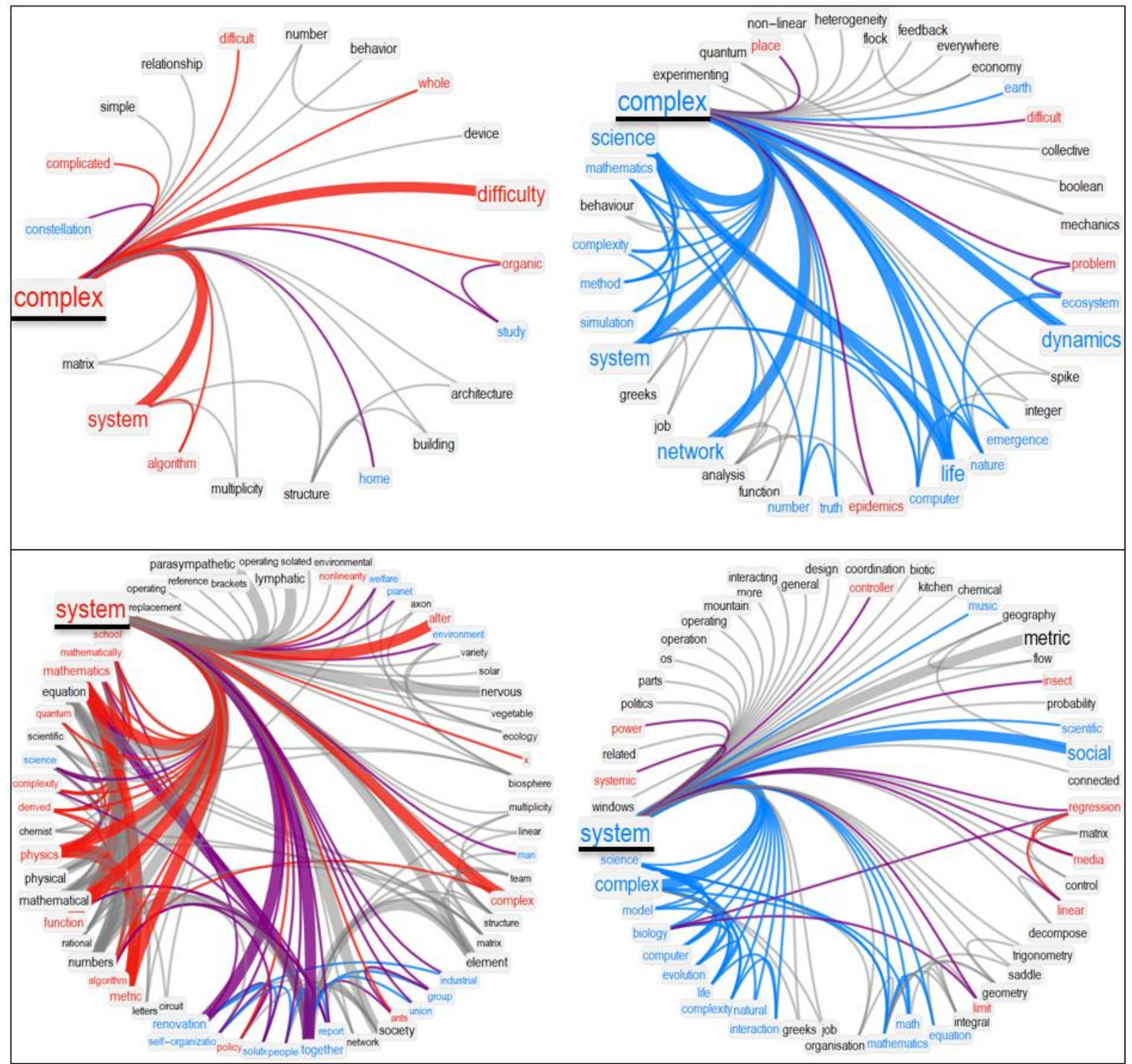

Figure 1. Local neighborhood of forma mentis networks for students (left) and researchers (right) around the key concepts "complex" (top) and "system" (bottom). These key concepts are underlined in black. Words with higher degrees, i.e. more links, have a larger font size. Negative concepts are highlighted in red while positive concepts are in cyan. Neutral concepts are in black. Stronger conceptual associations, repeated by at least two participants, are reported as thicker and their endpoints reported with a larger font size. Links between positive (negative) concepts are in cyan (red), whereas links between positive and negative concepts are in purple. Links between neutral concepts are in grey.

The perception that young researchers exhibited about "complex" was mostly positive, with many strong conceptual associations entwining aspects of complexity science like 
"emergence", "model", "simulation", "network", "life" and "dynamics". Importantly, these concepts were also identified as fundamental in the community-driven booklet "Complexity Explained" written by complexity scientists (De Domenico et al., 2019). Importantly, researchers were able to associate "complex" to "science", a link that was absent in the students' mindset and further indicating that high school students were not aware of complexity science. Despite this missing association, both these groups were able to link "complex" and "system", although their perceptions of the latter concept differed greatly.

The students' mental representation of "system" was dominated by biological concepts related to systems in the human body (e.g. "nervous", "lymphatic") usually studied at school. Another cluster associated to "system" was relative to mathematical and physical concepts like "equation" and "matrix", also commonly studied in school. Notice that most of the mathematical concepts associated to "system" were perceived as negative, as in past findings that reported a strong negative perception of quantitative STEM subjects cooccurring in students with a positive attitude towards abstract "science" (Stella et al., 2019). Students perceived "system" as a negative concept, surrounded it with a mixed negativeneutral aura but also associated it with a cluster of positive words like "society", "together" and "group". The "self-organisation" reported there was the Italian word "autoorganizzazione", which is related to student unions in Italy, and is not the same selforganisation observed in complex systems (Downey, 2018). Also, researchers provided positive associations between "system" and "social", but they also perceived "system" itself as a positive concept, surrounded by a mixed positive-neutral aura. The positive attitude in researchers' mindset around "system" was not widespread but rather concentrated in complexity topics like math tools and different STEM disciplines. This indicates a more concrete and global perception of "system" compared to that of the students. In the researchers' mindset, examples of systems were not limited to specific examples from biology or algorithms from mathematics, like in the students' FMN, but rather encompassed a variety of multiple disciplines and subjects. A clique analysis of the observed neighbourhoods is reported in Table 1 (researchers' FMN) and Table 2 (students' FMN). 
Table 1

Empirical cliques containing "complex" and "system": Researchers' FMN

\section{Cliques in the students' FMN} neighborhoods

\section{Observation probability (over configuration models)}

\begin{tabular}{|c|c|}
\hline \{analysis,complex,mathematics,number\} & 0.002 \\
\hline$\{$ complex,mathematics,method,science $\}$ & 0.005 \\
\hline \{complex,life,nature,science\} & 0.02 \\
\hline$\{$ complex,computer,nature,science\} & 0.02 \\
\hline$\{$ complex,complexity,science,system\} & 0.009 \\
\hline$\{$ complex,mathematics,science,system\} & 0.04 \\
\hline$\{$ complex,life,science,system\} & 0.04 \\
\hline$\{$ complex,computer,science,system\} & 0.036 \\
\hline \{biology,evolution,life,science,system\} & 0.0001 \\
\hline \{geometry,math,mathematics,system\} & 0.001 \\
\hline \{equation,integral,mathematics,system\} & 0.0002 \\
\hline$\{$ math,mathematics,science,system\} & 0.015 \\
\hline$\{$ computer,model,science,system $\}$ & 0.0006 \\
\hline$\{$ mathematics,organisation,science,system\} & 0.006 \\
\hline$\{$ complex,complexity,science,system\} & 0.01 \\
\hline$\{$ complex,mathematics,science,system\} & 0.04 \\
\hline$\{$ complex,life,science,system $\}$ & 0.02 \\
\hline$\{$ complex,computer,science,system\} & 0.01 \\
\hline
\end{tabular}

Note. This table shows empirical cliques containing either "complex" or "system" in the neighborhoods of these two concepts within the researchers' FMN. Only cliques with at least 4 nodes were considered. The probability of observing a clique in a sample of 5000 configuration models, preserving word degree but randomizing free associations, is reported on the right. Each neighborhood was randomized as a configuration model (see Methods).

All the observed cliques around "complex" and "system" can be observed in random null models with a probability lower than 0.05 . This result indicates that the detected cliques reflect cognitive associations that cannot be explained by network degree or random connections, see also Stella et al. (2019) for the interpretation of configuration models in linguistic networks.

Whereas researchers' free associations gave rise to several cliques with four elements around "complex", the reconstructed mindset of students featured no non-trivial clique (i.e. made by 4 elements or more). This is another quantitative indication that the students' perception around "complex" is semantically less rich and less clustered than the 
researchers' perception. The semantic content of the retrieved cliques indicates that researchers strongly connected "complex" with "mathematics" and mathematical jargon. This finding underlines the need for a mathematical jargon supporting complexity science, as pointed out also in other community driven approaches, see Cramer et al. (2018) and Complexity Explained.

Table 2

Empirical cliques containing "complex" and "system": Students' FMN

\begin{tabular}{|c|c|}
\hline Cliques in the students' FMN neighborhoods & $\begin{array}{c}\text { Observation } \\
\text { probability (over } \\
\text { configuration models) }\end{array}$ \\
\hline \{derived,physics,mathematics,mathematically,school,system\} & 0.00003 \\
\hline$\{$ algorithm,function,mathematics,report,system\} & 0.0001 \\
\hline \{physics,function,mathematics,rational,system\} & 0.001 \\
\hline \{derived,physics,function,mathematics,system\} & 0.0003 \\
\hline \{physics,mathematics,rational,science,system\} & 0.0005 \\
\hline \{physics,mathematics,mathematically,science,system\} & 0.0007 \\
\hline \{physics,mathematics,rational,scientific,system\} & 0.0002 \\
\hline \{equation,mathematical,numbers,rational,system\} & 0.00007 \\
\hline \{equation,mathematically,mathematical,numbers,system\} & 0.0002 \\
\hline \{equation,mathematics,numbers,rational,system\} & 0.0007 \\
\hline \{equation,mathematics,matrix,numbers,system\} & 0.0004 \\
\hline \{equation,mathematics,mathematically,numbers,system\} & 0.0004 \\
\hline$\{$ physics,mathematics,scientific,school,system\} & 0.0005 \\
\hline$\{$ element,together,system,team,structure $\}$ & 0.0001 \\
\hline$\{$ function,together,mathematics,report,system\} & 0.0008 \\
\hline$\{$ complexity,together,mathematics,numbers,system\} & 0.0007 \\
\hline \{together,mathematics,matrix,numbers,system\} & 0.003 \\
\hline \{together,mathematics,mathematically,numbers,system\} & 0.006 \\
\hline
\end{tabular}

Note. This table shows the empirical cliques containing "system" in its neighborhood in the students' FMN. The neighborhood of "complex" did not feature cliques with at least 4 elements in the students' FMN. Only cliques with more than 5 elements were considered. The probability of observing a clique in a sample of 5000 configuration models, preserving word degree but randomising free associations (right). Each neighborhood was randomized as a configuration model (see Methods). 
Interestingly, researchers clustered "complex" also with concepts from the life sciences and from the observation of the natural world, e.g. the clique \{"complex", "life", "nature", "science"\}. This richly clustered structure around "complex" was absent in the students' FMN. However, the students' mindset reported several cliques combining "system" with mathematical jargon, like "mathematics", "function" and "equation". As reported in Figure 1, though, the perceived sentiment dominating within these cliques was negative valence, which further indicated a clustered and distributed negative perception of mathematical concepts in students. One clique combines "complexity" with "numbers", suggesting again a connotation of complexity in relation with complex numbers in algebra. The strong predominance of mathematical jargon in the cliques surrounding "system" indicates that, differently from researchers, who reported concepts related to computers and nature's observation, students' perception of "system" was limited to a mathematical definition (e.g. of equations, etc.).

\section{Global comparison of associative mindsets represented by forma mentis networks}

Local comparisons between "complex" and "science" across researchers' and students' FMNs are informative of which associates/meanings were attributed to such concepts by any of the two groups. However, would it be possible to rank "complex" and "science" according to their centrality in the global network, including hundreds of different STEM concepts? The consensus translations implemented here and the filtering of free associations/links in terms of minimum spanning trees (i.e. the selection of links being included in the MST, cf. Methods) enabled a comparison of FMNs also at a global scale. A total of 928 common concepts were identified in both the researchers' and students' FMNs. Conceptual relevance was computed by considering closeness centrality of these 928 concepts over the minimum spanning tree connecting them (cf. Methods). Closeness captures semantic relatedness, so that a word with a higher closeness is, on average, more closely related to its connected concepts (Siew et al., 2019; Stella \& Zaytseva, 2020). In this way, ranking concepts according to their closeness is representative of their conceptual relevance in a suitably large networked mindset. Table 3 compares the top-10 words in highschoolers' and researchers' FMNs.

Table 3 shows that for both students and researchers, "science" was a highly central concept. That word was not used as a cue (Stella et al., 2019) but emerged from participants' associations. Its relevance in the FMNs is a confirmation that the cue words were STEMrelated and the methodology was able to elicit semantically related concepts. Researchers identified as central several different disciplines, like physics, chemistry and biology, 
whereas students focused their mindset more about physics-related topics and examrelated words, e.g. "grade" and "report".

\section{Table 3}

Top 10 concepts as ranked by closeness centrality in the minimum spanning tree: Students' and researchers' FMNs combined

\begin{tabular}{cccccc}
\hline \hline Rank & $\begin{array}{c}\text { Word for } \\
\text { Students }\end{array}$ & $\begin{array}{c}\text { Word for } \\
\text { Researchers }\end{array}$ & Rank & $\begin{array}{c}\text { Word for } \\
\text { Students }\end{array}$ & $\begin{array}{c}\text { Word for } \\
\text { Researchers }\end{array}$ \\
\hline $\mathbf{1}$ & approximation & physics & $\mathbf{6}$ & digit & science \\
$\mathbf{2}$ & physics & approximation & $\mathbf{7}$ & scientific & electricity \\
$\mathbf{3}$ & grade & ecosystem & $\mathbf{8}$ & interval & university \\
$\mathbf{4}$ & science & current & $\mathbf{9}$ & simplification & biology \\
$\mathbf{5}$ & report & chemistry & $\mathbf{1 0}$ & about & space \\
\hline \hline
\end{tabular}

"Complex" was peripheral in the filtered students' mindset (rank \#87) and more central in the researchers' mindset $\left(\#_{31}\right)$, indicating a stronger conceptual relatedness between "complex" and other scientific concepts within the mental organisation of knowledge represented by the researchers' FMN than in students'. This strong difference confirms the above local findings about students displaying almost no awareness of complexity science in comparison to researchers.

"System" was ranked higher in the students' FMN (\#13) and lower by researchers (\#111), who nonetheless ranked concepts related to complexity science like "theory" and "science" higher than system itself (\#12 and \#6, respectively).

Despite microscopic differences, the two rankings highlighted by spanning trees were positively correlated, as indicated by a Spearman Rank test $(r=0.2741, p<0.001)$. This correlation indicates that students and researchers weakly tended to allocate conceptual centrality to the same words, on average. Examples of such tendency are "science", "approximation" and "physics", which were equally ranked in the top 10 by both groups.

\section{DISCUSSION}

This report reconstructed and compared high school students' and young complexity researchers' mindsets around "complex" and "system" through forma mentis networks (Stella et al., 2019; Stella 2020a; Stella \& Zaytseva, 2020). High schoolers displayed basically 
no awareness of complexity science, focusing their perception of "complex" around words expressing meanings of difficulty, complicatedness and negatively perceived mathematical jargon (e.g. links like "complex" and "number"). Instead, researchers perceived "complex" as a contextually richer concept, better connected with key aspects of complexity science as identified also in a recent work (De Domenico et al., 2019), such as "networks", "emergence", "simulation" and "dynamics". Also, for the perception of "system", researchers reported associations spanning multiple STEM subjects, whereas students mainly associated "system" with biological examples and mathematical jargon. Both groups reported a strong link between "complex" and "system", though in students this connection was more in the context of mathematical equations. In both groups, a social component of "system" was represented by conceptual links to society and social systems.

Differences in the semantic relevance of "complex" in the global students' and researches' mindsets further corroborated the above local patterns in indicating that highschool students were not able to express awareness of complexity science and mentally reconstructed "complex" in terms of complicatedness and difficulty. An alternative interpretation of the fragmented associations reported here would be for students to having been exposed to complexity knowledge, but not possessing the language necessary for expressing awareness of it, as recently discussed by Brown (2019). In any case, students did not exhibit any awareness of complexity science, unlike the researchers sampled in the study. This difference could be attributed to differences in expertise, with experts having more training and knowledge. Nonetheless, the students of this dataset studied a wide variety of STEM topics simultaneously, thanks to their educational curriculum, i.e. "liceo scientifico". More in detail, topics like calculus, probability, statistics, physics, biology, chemistry, computer science and philosophy were all simultaneously studied by each student. Compared to researchers in single-discipline departments, students had the exceptional and unique opportunity to access multiple scientific disciplines at the same time. And yet, despite this multidisciplinary exposure, no bridges or references to complexity science were traced here in the students' mental construct representing complex systems. This gap has to be interpreted as an effect of a "silos education", where different disciplines are studied in detail but with no connections or applications to the real world (Donina et al., 2017). A 'silos' mindset hampers knowledge creation and diffusion, with negative implications also over innovation and management. Detecting and overcoming "silos education" is therefore a key challenge for future education (Donina et al., 2017; Cramer et al., 2018; Van der Cingel 2018) and FMNs might play a key role in identifying it in student populations (Stella \& Zaytseva, 2020). Another prominent difference was in emotional attitudes: Students perceived "complex" and "system" as negative concepts, surrounded by a mixed negative-neutral emotional aura, whereas researchers reported a considerably more positive perception, arising also from those 
concepts and subjects perceived negatively by students (e.g. "maths" and "physics"). Improvements in these negative attitudes could be achieved by implementing teaching strategies aiming at a deeper understanding of complexity, like problem-based learning (Lizier et al., 2018) or the adoption of peer-oriented social learning, which was shown to improve learning outcomes in physics (Koponen et al., 2017).

Forma mentis networks can highlight correlations between stances but not provide causal links, so that it is not possible to determine here whether the positive perceptions of "complex" and "system" observed in researchers were influenced by the positive stances towards "maths" and "physics" or vice versa. Despite this limitation, the current investigation highlighted the simultaneous presence of positive perceptions towards STEM subjects when linked together to complexity science transdisciplinary topics, as in the mindset of researchers. This suggests the possibility of restoring positive attitudes towards "complex" and "system" in high school students while also promoting a positive, interconnected and transdisciplinary perception of STEM subjects, which reportedly improved learning outcomes in previous teaching activities (Cramer et al., 2018; Van der Cingel 2018; Lizier et al., 2018).

The results reported in this research report indicate both an urgent need and a possibility. The need originates from the negative and incomplete perceptions of complexity and mathematical disciplines (Stella et al., 2019; Stella 2020a) among students who are on the verge of choosing their professional education. The negative auras of the above concepts, concentrating negative associations, indicate that students perceive anxiety and stress when thinking of "complex", "system" and also mathematical jargon (cf. Stella et al., 2019). Reducing the negative perception of "complex" and "system" requires building positive associations, but which ones? Inspiration could be drawn from the mindset postgraduate researchers, mastering $\mathrm{PhD}$-level skills specific of complexity science and sharing a passion over complexity science. Adopting the mindset of these researchers as inspiration would offer a potentially beneficial possibility for improving the students' perception of mathematical concepts too, given the mental associations reported here and in previous studies (Stella et al., 2019; Stella, 2020a; Stella \& Zaytseva, 2020). In this specific, data-informed way, promoting a better understanding of complexity science would be key to outreach activities or teaching activities aiming at improving the students' positive attitude towards STEM subjects.

The current analysis was based on the mindset of students already in the crucial moment of choosing their future educational path at the university level first and on the job market later. Considering the criticalities outlined here in terms of an anxious perception of mathematical and complexity-related concepts, due interventions should be devoted way before this stage, potentially at the start of high school or as soon as basic mathematical tools have been already acquired by students (Lizier et al., 2018). Several 
promising approaches promoting the culture of complexity science have already been developed and are currently being tested, in a synergistic approach between education researchers, complexity scientists, teachers and students (cf. Cramer et al., 2018; Lizier et al., 2018).

Forma mentis networks can be powerful tools for the identification and planning of outreach activities, since they can extract detailed information about how different groups or even individuals structure their mindsets around a topic. For instance, building the FMN of a class before the start of a professional experience, analogously to what was done in Stella and Zaytseva (2020), can provide knowledge about negative or critical perceptions that might be present in a cohort of students and impact learning. Differently from standard surveys, FMNs can monitor explicit and implicit perceptions without the strict requirement of designing direct questions. The free association task, in fact, can detect implicit biases through associated words, e.g. the cue "math" eliciting the association of "anxiety" in relation to an implicit math anxiety, see Stella (2020a) for more details.

The main limit of FMNs is in their need to gather empirical data from human participants through controlled experiments in order to obtain densely connected networks. The task required for building the networks used here lasted for approximately 20 minutes. Despite the need for brief lab experiments, forma mentis networks are quite flexible, as they do not limit stance detection to confined, hard questions but let conceptual stances emerge through free associations and valence identification. Another way of extracting FMNs from text has been successfully shown to identify relevant concepts in structured mindsets (Stella, 202ob), at the cost of losing the ability to detect implicit knowledge or associations.

FMNs can be adopted in different ways for educational purposes. For instance, different FMNs for the same population could be built for tracking the progress or changes in mindsets over time before and after an educational intervention, analogously to the approach of Stella and Zaytseva (2020), who reported the formation of more positive, concrete and better structured mindsets in college students enrolled in a summer job. Additional research is necessary to understand and quantify the costs and academic impact of establishing specific conceptual associations through aimed teaching interventions. A promising modelling approach would be the application of some aspect of dynamical systems theory (Koopmans 2011) to forma mentis networks, providing a statistical reference to the likelihood of potential changes in perception in terms of catastrophic phase transitions.

The differences reported here between complexity researchers and students might be used also for reverse-engineering an automatic classifier of domain expertise. In agreement with pioneering approaches using cognitive network science (Siew 2019, Siew et al., 2019) or concept maps (Tyumeneya et al., 2017; Koponen et al., 2017) for identifying domain 
expertise, the structure of forma mentis networks could be used for defining and predicting the acquisition of a rich complex systems mindset thanks to their combination of emotional/sentiment patterns and semantic associations.

\section{ACKNOWLEDGEMENTS}

The author acknowledges the Scientific Committee of the Complex Forma Mentis project (http://www.complexmentis.com/, Last Accessed: 17/12/2019).

\section{REFERENCES}

Aitchison, J. (2012). Words in the mind: An introduction to the mental lexicon. Wiley \& Sons.

Brown, S. L. (2019) A patterning approach to complexity thinking and understanding for students: A case study. Northeast Journal of Complex Systems (NEJCS), 1, 6. http://dx.doi.org/10.22191/nejcs/voli/iss1/6

Cramer, C. B., Porter, M. A., Sayama, H., Sheetz, L., \& Uzzo, S. M. (Eds.). (2018). Network science in education: Transformational approaches in teaching and learning. Springer.

De Deyne, S., Navarro, D. J., \& Storms, G. (2013). Better explanations of lexical and semantic cognition using networks derived from continued rather than single-word associations. Behavior Research Methods, 45, 480-498. http://dx.doi.org/10.3758/s13428-012-026o-7

De Domenico, D. Brockmann, C. Camargo, C. Gershenson, D. Goldsmith, S. Jeschonnek, L. Kay, S. Nichele, J.R. Nicolás, T. Schmickl, M. Stella, J. Brandoff, A.J. Martínez Salinas, H. Sayama. (2019). Complexity Explained. http://dx.doi.org/10.17605/osf.io/tqgnw

Donina, D., Seeber, M., \& Paleari, S. (2017). Inconsistencies in the governance of interdisciplinarity: the case of the Italian higher education system. Science and Public Policy, 44, 865-875. https://doi.org/10.1093/scipol/scxo19

Downey, A. (2018). Think complexity: Complexity science and computational modeling. O'Reilly Media.

Flament, C. (2017). Théorie des graphes et structures sociales (Vol. 2). Walter de Gruyter \& Co.

Koopmans, M. (2011). Epilogue: Psychology at the edge of chaos. In S. J. Guastello, M. Koopmans, \& D. Pincus (Eds.) Chaos and complexity in psychology: The theory of 
nonlinear dynamical systems (pp. 506-526). Cambridge University Press. http://dx.doi.org/10.1017/CBO9781139058544.018

Koponen, I. T., Kokkonen, T., \& Nousiainen, M. (2017). Modelling sociocognitive aspects of students' learning. Physica A: Statistical Mechanics and its Applications, 470, 68-81. https://doi.org/10.1016/j.physa.2016.11.139

Lizier, J. T., Harré, M. S., Mitchell, M., DeDeo, S., Finn, C., Lindgren, K., Lizier, A. L., \& Sayama, H. (2018). An interview-based study of pioneering experiences in teaching and learning complex systems in higher education. Complexity. http://dx.doi.org/10.1155/2018/7306871

Morin, E. (2007). Restricted complexity, general complexity. In Science and us: Philosophy and complexity (pp.1-25). World Scientific.

Newman, M. (2018). Networks. Oxford University Press.

Papageorgiou, G., Stamovlasis, D., \& Johnson, P. M. (2010). Primary teachers' particle ideas and explanations of physical phenomena: Effect of an in-service training course. International Journal of Science Education, 32, 629-652. https://doi.org/10.1080/09500690902738016

Posner, J., Russell, J. A., \& Peterson, B. S. (2005). The circumplex model of affect: An integrative approach to affective neuroscience, cognitive development, and psychopathology. Development and Psychopathology, 17, 715-734. http://dx.doi.org/10.1017/So954579405050340

Siew, C. S. (2019). Using network science to analyze concept maps of psychology undergraduates. Applied Cognitive Psychology, 33, 662-668. https://doi.org/10.1002/acp.3484

Siew, C. S., Wulff, D. U., Beckage, N. M., \& Kenett, Y. N. (2019). Cognitive network science: A review of research on cognition through the lens of network representations, processes, and dynamics. Complexity. https://doi.org/10.1155/2019/2108423

Stella, M., De Nigris, S., Aloric, A., \& Siew, C. S. (2019). Forma mentis networks quantify crucial differences in STEM perception between students and experts. PloS one, 14(10). https://doi.org/10.1371/journal.pone.0222870

Stella, M. (2020a). Forma mentis networks reconstruct how Italian high schoolers and international STEM experts perceive teachers, students, scientists, and school. Education Sciences, 10, 17. https://doi.org/10.3390/educsci10010017

Stella, M. (202ob). Text-mining forma mentis networks reconstruct public perception of the STEM gender gap in social media. arXiv preprint arXiv:2003.08835. 
Stella, M., \& Zaytseva, A. (2020). Forma mentis networks map how nursing and engineering students enhance their mindsets about innovation and health during professional growth. PeerJ Computer Science, 6, e255. https://doi.org/10.7717/peerj-cs.255

Tyumeneva, Y., Kapuza, A., \& Vergeles, K. (2017). Distinctive Ability of Concept Maps for Assessing Levels of Competence. Pilot study. Educational Studies, 4, 150-170.

Van der Cingel, P. (2018). How to educate navigators in a complex world: Making a case in higher professional education in the Netherlands. Complexity, Governance and Networks, 4(1). 